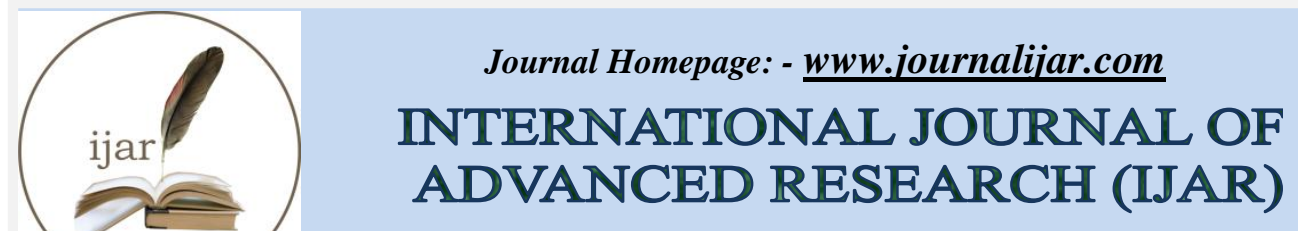

Article DOI: 10.21474/IJAR01/2876

ISSN NO. 2320-5407
DOI URL: http://dx.doi.org/10.21474/IJAR01/2876

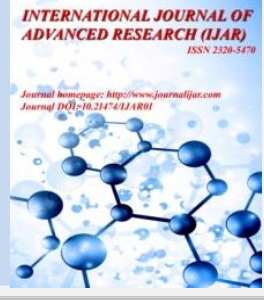

RESEARCH ARTICLE

\title{
METASTATIC OVARIAN TUMOR DISCOVERED ACCIDENTALLY DURING CESAREAN SECTION IN A 34-YEAR-OLD WOMAN: A CASE REPORT.
}

\author{
Ghada Esheba $^{1,2}$, Wafa'a Al-Harbi ${ }^{2,3}$, GhufranKheshaifaty ${ }^{2,3}$, Kholoud Al-Harbi ${ }^{2,3}$, Ala'a Al-Orabi ${ }^{2,3}$ and \\ Moayad Turkistani ${ }^{2,3}$. \\ 1. Associate Professor of Pathology;Department of Pathology, Faculty of Medicine, Tanta University, Egypt. \\ 2. Department of Pathology, Faculty of Medicine, Umm Al-Qura University, KSA. \\ 3. Intern; Faculty of Medicine, Umm Al-Qura University, KSA.
}

\section{Manuscript Info}

\section{Manuscript History}

Received: 21 November 2016

Final Accepted: 21 December 2016

Published: January 2017

\section{Key words:-}

Primary ovarian tumors, krukenburg tumor, CK7, CK20, CDX-2, luteoma of pregnancy.

\section{Abstract}

Krukenberg tumor is a rare metastatic ovarian carcinoma usually occur in female between 30-40-year-old and rarely seen after menopause. Stomach is the most common primary site.

Histopathological features of krukenberg tumors appears as diffuse stromal proliferation, mucus-production, and numerous signet-cells and these tumors spread mostly by lymphatic route. Treatment and prognostic factors are not well established.

This study describes a 34-year-old female with a unilateral ovarian mass discovered accidentally during cesarean section delivery and it was misdiagnosed as luteoma of pregnancy, but histopathological examination showed a diffuse infiltration of the ovary and omentum by malignant cells, some of which have signet ring morphology. These findings were not correlated with luteoma of pregnancy or any other types of primary ovarian tumors like primary surface epithelial tumor, sex cord stromal tumor or germ cell tumor. A panel of immunohistochemicalmarkers were ordered and the results were as follow: (negative CK7, AFP, and alpha inhibin, and positive EMA, CK20 and CDX-2). these findingswere diagnostic for metastatic adenocarcinoma either from the stomach or pancreas (krukenberg tumor). Two weeks later, the patient was evaluated and a large gastric tumor was found in her stomach and she underwent gastrectomy.

Copy Right, IJAR, 2016,. All rights reserved.

Corresponding Author:-GhadaEsheba.

Address:-Associate Professor of Pathology; Department of Pathology, Faculty of Medicine, Tanta University, Egypt. Department of Pathology, Faculty of Medicine, Umm Al-Qura University,KSA. 


\section{Introduction: -}

Ovarian cancer is the second most common cancer among all gynecological malignancies and it's one of the leading causes of cancer death among women. Malignant ovarian lesions include primary lesions that develop from the ovarian tissue itself or secondary lesions due to ovarian metastasis from primary lesion elsewhere. ${ }^{[1]}$

Primary ovarian tumors include surface epithelial stromal tumors, sex-cord stromal tumors, germ cell tumors and other more rare types. Ovarian epithelial tumors account for the majority of all ovarian tumors. And they are classified according to histological cell type into serous, mucinous (formed by cells of the endocervical epithelium or those of the intestinal epithelium), endometrioid (formed by cells of the internal lining of the endometrium) clear and transitional tumors. These tumors usually range from being benign to borderline or malignant tumor. ${ }^{[1]}$

Krukenberg tumors are rare secondary ovarian cancer due to metastatic adenocarcinoma of the gastrointestinal tract, mainly originate from the stomach. It is bilateral in most of the cases. They are rarely discovered during pregnancy, thus, possess diagnostic challenges to the physicians since its usual symptoms can be attributed to pregnancy. Local extension of the tumor produces gastrointestinal symptoms which could be confused with the effect of pregnancy hormones on the gastrointestinal tract or misdiagnosed with hyperemesis gravidarum. Abdominal distention can also be masked by the gravid uterus. Furthermore, new onset ovarian masses discovered during pregnancy or delivery can be mistaken with pregnancy luteoma, which is, benign ovarian neoplasm that is usually asymptomatic and discovered incidentally during surgeries or imaging. ${ }^{[2]}$

These metastatic tumors usually occur in age group between 30-40 years and rarely seen after menopause. Diagnosis of krukenberg tumors made histologically by the presence of diffuse stromal proliferation, mucus-production and neoplastic signet ring cells. ${ }^{[3]}$

The prognosis of patients with this type of tumor are extremely poor, median survival of 7-14 months.

Treatment guidelines has not been established well because of rarity of the disease. And still there is no optimal therapy for this tumor. ${ }^{[4]}$

\section{Case presentation: -}

A 34-year-old pregnant woman on her usual state of health came to the hospital forcesarean delivery. During C/S, her surgeon accidentally discovered a unilateral ovarian mass.Thereafter, oophorectomy and partial omentectomy were performed. The specimen was sent for further evaluation.

The patient did not report any complaint before, and her family history was noncontributory.

\section{Pathological findings: -}

Gross examination revealed a $12 \mathrm{X} 7 \mathrm{~cm}$ tumor with smooth external surface and there were no nodules. The cut section showed fleshy whitish tissue with central areas of necrosis and hemorrhage. The microscopic examination showed a diffuse infiltration of the ovary and omentum by signet ring cells, which contain mucin that pushed the nucleus against cell membrane. (Fig.1,2). These findings were not correlated with luteoma of pregnancy or any primary ovarian tumors like surface epithelial tumor, sex cord stromal cell tumor or germ cell tumor but these findings were suspicious for metastatic krukenberg and adenocarcinoma.

Therefore, we ordered a panel of immunohistochemical markers to reach the definitive diagnosis.

The results of immunohistochemical staining for tumor cells were as follow: positive for CK7, CK20 Fig.3(A), CDX-2 and CD99 (Diffuse immunoreactivity). EMA was also positive Fig.3(B). At the same time, tumor cells staining negative for AFP, S-100, HMB-45 and NSE. Inhibin was completely negative.

Further investigations were done to identify the primary site of the tumor and after two weeks of extensive work upfor this patient; the primary tumor was found in stomach and it was a gastric adenocarcinoma. 

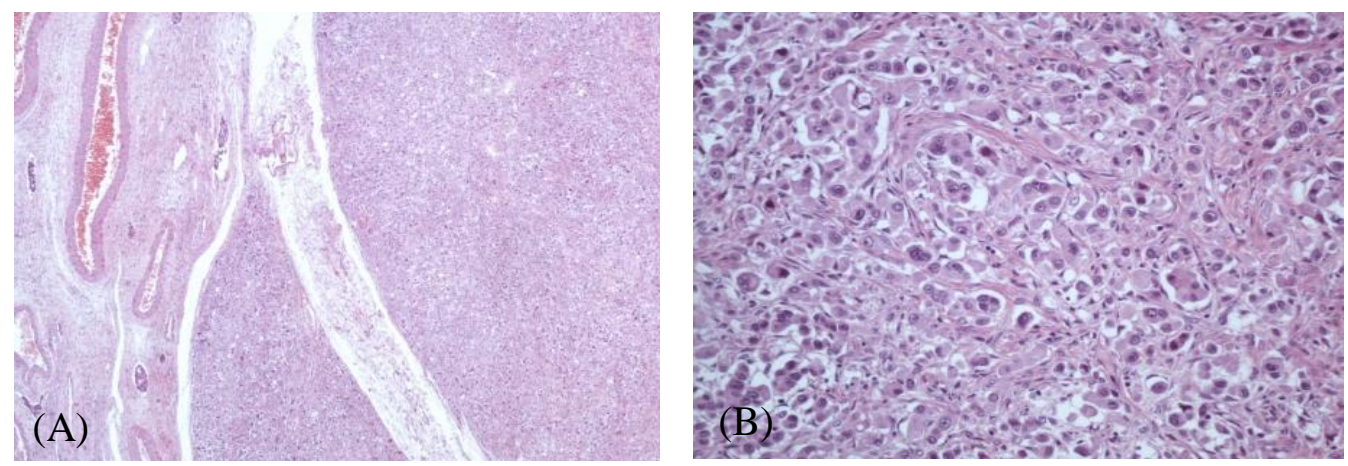

Fig.1:- Diffuse infiltration of ovarian stroma by malignant cells arranged singly and in small aggregates, some of these cells have vacuolated cytoplasm (signet ring appearance)

Finally, our patient underwent gastrectomy.

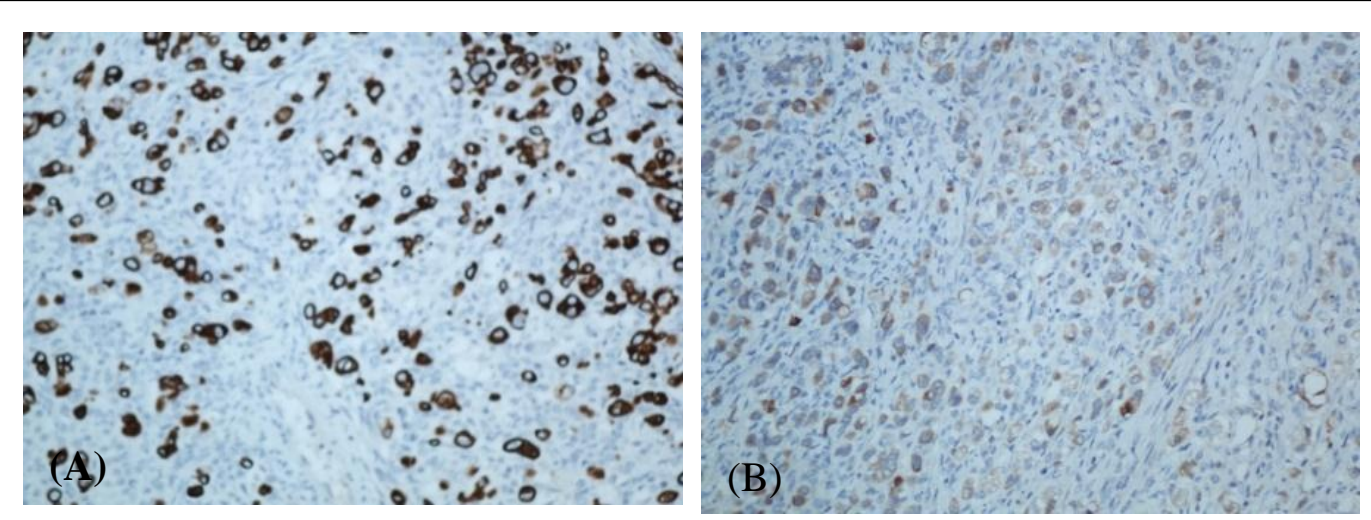

Fig.3:- (A) positive CK20. (B) positive EMA.

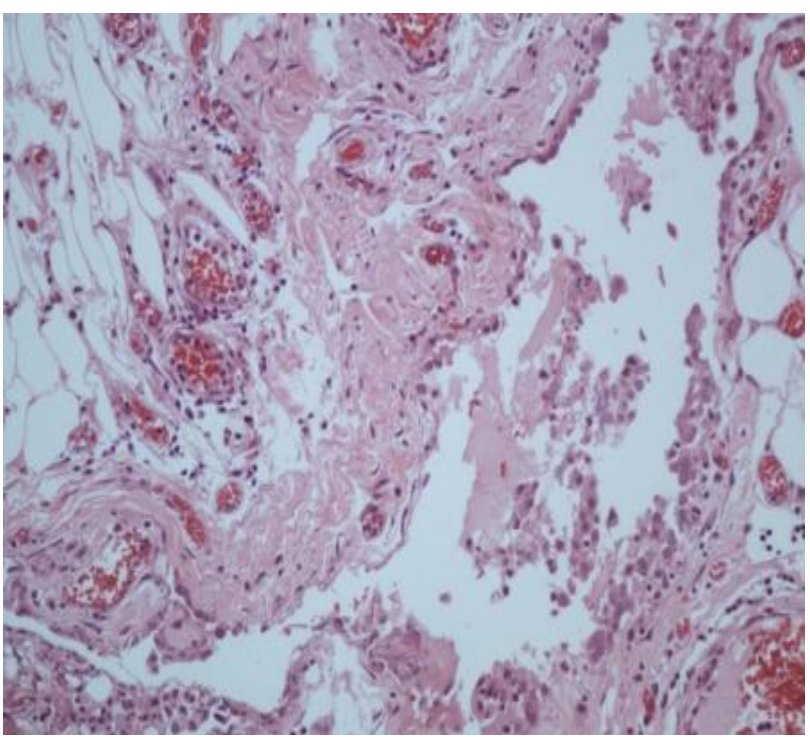

Fig.2:-The omentum showed diffuse multiple signet ring cells. 


\section{Discussion: -}

Metastases of mucin-secreting signet ring cell cancer (SRCC) to ovarian tissues are known as Krukenberg tumor, which is uncommon metastatic ovarian cancer originate from gastrointestinal tract. Stomach is the most common primary site, followed by colon, appendix, and breast cancer. Usually occur in age group between 30-40 year old. ${ }^{[5]}$ as in this case, she is a 34-year old.

Krukenberg tumor rarely occurs during pregnancy, diagnosis sometimes is difficult as the presenting symptoms are usually attributed to the pregnancy and there is a maternal and fetal risk for invasive workup.

In most of cases, primary tumor and its metastasis to ovary are diagnosed at the same time, but in 20-30\% of cases, ovarian metastasis diagnosed after primary tumor resection. ${ }^{[6]}$

It has been reported that $80 \%$ of metastatic ovarian carcinomas are bilateral ${ }^{[7]}$ and mostovarian carcinomas that have beenmetastasizedfrom primary tumors of the large intestine shows solid and cystic components. ${ }^{[8]}$

Our case is parallel to this experience, since the patient had a unilateral metastatic ovarian tumor and the diagnosis of primary gastric adenocarcinoma was established after 2 week of accidental discovery of metastatic krukenburg tumor.

At the beginning, the ovarian mass in our case was misdiagnosed as luteoma of pregnancy which is a non-neoplastic hormonal dependent lesion of the ovary arises from pre-existing luteinized stromal cells, usually discovered incidentally during $\mathrm{C} / \mathrm{S}$ or postpartum tubal ligation, regress spontaneously postpartum unless there's indication of surgery and it needs accurate diagnosis as it maybemisdiagnosed as malignant ovarian tumor which results in unnecessary surgery. ${ }^{[2]}$

In our case, the histopathological findings were not consistent with the diagnosis of pregnancy luteoma as it shows diffuse infiltration of the ovary and omentum by malignant cells that produce excessive mucin intracellularly. The mucin forms large vacuole within the cells and pushes the nucleus from the center to the periphery against the cell membrane leading to the characteristic feature called "signet ring appearance".

Several mechanisms of metastasis have been reported, lymphatic is the most common route of metastasis, followed by hematogenous spread, direct invasion and peritoneal seeding. ${ }^{[6]}$

There are only 50 cases of Krukenberg tumors occurring during pregnancy have been reported in the literature and there was no significant impact on fetal survival as most (97\%) of the reported cases have led to the delivery of healthy baby. ${ }^{[9]}$

Symptomatic patients usually present with abdominal pain, distention, vomiting and weight loss. Virilization and hirsutism may occur due to an effect of estrogen and androgen hormones, other gynecological symptoms such as amenorrhea, menstrual irregularity, and vaginal bleeding are much less frequent in the literature cases. ${ }^{[6]}$ The pseudo-Meig syndrome is a rarely associated condition, which is malignant ovarian tumor with benign hydrothorax and ascites. ${ }^{[4]}$

Here, we present an unusual case of Krukenberg tumor which discovered accidently during C/S delivery, not preceded by any significant symptoms.

Sometime it is difficult to differentiate morphologically between primary signet ring cell carcinoma of the ovaries and Krukenberg tumors, because usually they are resembling to each other.

Immunohistochemistry is a valuable diagnostic tool in differentiating between them. primary ovarian surface epithelial tumors are mostly positive to CK7 and negative to CK20 and CDX-2. By contrast, metastatic adenocarcinoma is less frequently to be positive for CK7 but is positive for CK20 and CDX-2 in most cases.

Park et al23 found that 71\% (207 of 289) of the gastric carcinomas stained positively for CK7, whereas only 9\% (21 of 225) of the colorectal carcinomas proved to be CK7 positive, and 41\% (117 of 89) of the gastric carcinomas and $73 \%$ (165 of 225) of the colorectal carcinomas were CK20 positive. ${ }^{[4]}$ 
Our patient's tumor stained positively for both CK7 and CK20, indicative of gastric origin.

The treatment strategy is controversial in Krukenberg tumor. surgical resection and aggressive chemotherapy can possibly improve the outcome. ${ }^{[5]}$ In advanced disease, palliative treatment (Chemotherapy and Radiotherapy) is considered. ${ }^{[6]}$

The prognosis is poor, the median overall survival time is 2 years. The prognostic factors still not well established. One study reported that, regardless of cancer stage patients who underwent metastectomy and chemotherapy had better prognosis than those who are received chemotherapy alone. ${ }^{[10]}$

Survival of patients who underwent both metastasectomy and gastrectomy was longer than the patients who underwent metastasectomy only.Gastrectomy and absence of ascites are considered as risk factors associated with good survival rate. Inability of resection for the primary tumor is indicate a poor survival rate. ${ }^{[10]}$

Rarely, metastatic ovarian tumors are discovered before primary site, If the primary tumor is identified after the discovery of secondary tumor the prognosis is poor. ${ }^{[5]}$ As in our case.

CA 125 level normally elevated during pregnancy and immediately after delivery. However, markedly elevation is commonly seen during cancer ${ }^{[11]} \mathrm{CA} 125$ levels are considered as a useful prognostic marker in evaluating patient's outcome after tumor resection. ${ }^{[5]}$ Patient's age and size of ovarian tumorhas no role in prognosis. ${ }^{[4]}$

\section{Conclusion:-}

Krukenbuerg tumors are uncommon metastatic gastrointestinal cancer to the ovary mostly from the stomach, presenting with vague symptoms, radiological imaging, histological findings and immunohistochemical evaluation aid in diagnosis and identification of the primary malignancy.

Unfortunately, there are limited treatment options and no optimal treatment has been established. It has a poor prognosis and short median survival time. However, better outcome depends on early diagnosis. As in our case synchronous diagnosis and treatment of the primary cancer and its metastatic tumor along with regular follow-up can prolong the survival time.

Leuteoma of pregnancy should be considered as a differential diagnosis for any ovarian mass diagnosed during pregnancy to avoid unnecessary surgery, the former lesion regress spontaneously in postpartum period.

We report this case because of the relatively rarity of accidentally discovered metastatic ovarian tumor from gastric adenocarcinoma during CS delivery which waspreceded by a normal full-term pregnancy.

\section{References:-}

1. Chen V.W., Ruiz B., Killeen J.L., et al., Pathology and classification of ovarian tumors. CNCR Cancer, 2003. 97(S10): p. 2631-2642.

2. Nanda, A., U.A. Gokhale, and G.R. Pillai, Bilateral pregnancy luteoma: A case report. Oman Med. J. Oman Medical Journal, 2014. 29(5): p. 37.372-1

3. Fang WU, Xiaoaizhao, Baibing MI et al., Clinical characteristics and prognostic analysis of Krukenberg tumor. mol clin onc Molecular and Clinical Oncology, 2015.

4. Rawasia W.F., Chaddha U., El-Kersh K., et al.,Dyspnoea and the ovaries: a rare presentation of Krukenberg tumour. BMJ case reports, 2013. 2013.

5. Umakanthan, S., M.M. Bukelo, and K. Hardik, A 36-year-old female with Krukenberg tumor from a colonic carcinoma. Journal of cancer research and therapeutics, 2015. 11.(4)

6. Sahin S., Karatas F., Hacioglu B., et al.,Krukenberg tumor presenting with amenorrhea as the sole initial symptom: Case report and review of the literature. Journal of cancer research and therapeutics, 2015. 11.(4)

7. AL-AGHA OM, \& NICASTRI AD. (2006). An in-depth look at Krukenberg tumor: an overview. Archives of Pathology \& LaboratoryMedicine. 130, 1725-30. 
8. Shiono S., Saito T., Fujii H., et al., A case of Krukenberg carcinoma metastasized from colon cancer resembling mucinous cystadenocarcinoma of the ovary. 2013, e-Century Publishing Corporation.

9. Goyal S, Bhankar H, Tyagi N., et al., Incidentally diagnosed Krukenberg tumor in pregnancy: A rare presentation with dismal outcome. Clinical Cancer Investigation Journal, 2016;5(2):196.

10. Kumar M., Kumar A., Maroules M., et al.,Postmenopausal vaginal bleeding as initial presentation of gastric cancer: a case report with literature review of prognostic factors and treatment of krukenberg tumor. Annals of translational medicine, 2016. 4.(4)

11. Co P.V., Gupta A., Attar B.M., et al.Gastric cancer presenting as a krukenberg tumor at 22 weeks gestation. J. Gastric Cancer Journal of Gastric Cancer, 2014. 14(4): p. 275-278. 